\title{
Género, tenencia y bosques comunitarios en Uganda
}

\author{
Abwoli Y. Banana', Mohamed Bukenya', Eusobio Arinaitwe², Betty Birabwa' y Sylvester Ssekindi'
}

\section{Mensajes clave}

- A pesar de que las comunidades tienen mayor participación en el manejo forestal en Uganda, las mujeres han sido en gran parte excluidas de la toma de decisiones.

- Es necesario que más mujeres asuman posiciones de liderazgo a fin de que puedan definir mejor la agenda.

- Los grupos de usuarios forestales formales (a diferencia de los informales) parecerían aumentar la calidad y el nivel de participación de las mujeres en la toma de decisiones relacionada con el manejo forestal.

- Una mejor información y recursos financieros podrían ayudar a las mujeres a participar más efectivamente en el manejo de los recursos forestales en el país.

- Las mujeres representaron solo el 5\% de las posiciones de liderazgo político y técnico en los tres distritos estudiados. Existe una clara necesidad de establecer medidas de acción afirmativa.

- El personal técnico y los líderes políticos a nivel de distrito y subdistrito necesitan ser capacitados en cuestiones de género.

- Las mujeres que tienen cierto nivel de educación no son frecuentemente las mejores representantes de las necesidades de las mujeres: su estilo de vida, de la elite urbana, es muy diferente al estilo de vida de las mujeres rurales pobres.

\section{Introducción}

A pesar de la retórica legal y política que promete prestar más atención a las cuestiones de género en el manejo forestal, las mujeres continúan siendo marginadas de la toma de decisiones, incluyendo el manejo forestal colaborativo (MFC) y las reuniones de los grupos de usuarios forestales en Uganda (Obua et al. 1998 y Kugonza et al. 2009). Las normas sociales, la división del trabajo y las normas de conducta por género, así como los espacios y especies por género, restringen la participación de las mujeres en la toma de decisiones y en el manejo forestal (Howard y Nabanoga 2007). Estas normas sociales y prácticas culturales limitan la visibilidad, movilidad y conducta de las mujeres, aun cuando son miembros de grupos formales o informales (Mwangi et al. 2011).

El presente estudio evalúa el nivel de participación de las mujeres en la toma de decisiones, la definición de la agenda y la distribución de beneficios en múltiples niveles de gobernanza forestal en tres distritos de Uganda. Analiza el apoyo de actores externos a la participación de las mujeres en el manejo forestal comunitario. Finalmente propone modalidades para fomentar la inclusión de las mujeres en la toma de decisiones relacionada con los bosques.

La investigación se centró en los distritos de Mpigi, Masaka y Rakai, que forman parte de la zona agroecológica del lago Victoria y se caracterizan por sus reservas forestales centrales y locales, así como bosques privados y comunales/sagrados (Figura 1). Varias fuerzas impulsan la deforestación en el área de estudio: instituciones de gobernanza forestal débiles, una demanda de productos forestales alta (debido a una creciente población) y una implementación deficiente de las políticas gubernamentales en el sector del medio ambiente (Banana et al. 2010).

Además de estar bajo diferentes regímenes de tenencia, estos distritos se caracterizan por contar con una serie de actores externos que tienen como objetivo el empoderamiento de las comunidades locales en el manejo forestal y, en términos más generales, en el desarrollo. En este contexto, analizamos el nivel de importancia que el género ocupa en organizaciones y programas gubernamentales (del gobierno central) y no gubernamentales, en las comunidades forestales y en los consejos/ comités de los gobiernos locales que toman decisiones sobre el manejo forestal y asignan presupuestos.

\section{El género en organizaciones y programas}

La mayor parte de las organizaciones y los programas estudiados no están específicamente involucrados en el manejo forestal, sino que más bien trabajan con comunidades para mejorar la seguridad alimentaria, a través de una mejor conservación del suelo y el agua, así como las tecnologías de energía. El 75 \% de estas organizaciones había diseñado una política de género pero menos de un tercio reportó haber tenido éxito incorporando este tema.

Gran parte de las organizaciones dedicadas específicamente a los temas forestales son empresas del sector privado y están involucradas en el cultivo de árboles a gran escala, o entidades públicas que tienen a su cargo la administración de las reservas forestales del Gobierno, tanto locales como nacionales. En gran parte, los arboricultores privados no tenían interés en promover la participación local en el manejo forestal o analizar los roles de género. A pesar de tener la obligación legal de hacerlo, las entidades del gobierno involucradas en el manejo forestal no incluyen el género de forma prominente en sus proyectos y programas.

Solo una iniciativa gubernamental, el Proyecto para la Mejora de los Ingresos Agrícolas y la Conservación de los Bosques (FIECOC por sus siglas en inglés), promueve la participación de las mujeres en el cultivo de árboles en fincas. Aun así, las mujeres solo representan el 30\% de los individuos que solicitan ser miembros de FIEFOC. Este bajo nivel de participación se atribuye principalmente a las desigualdades de género en la propiedad de la tierra, las normas y prácticas culturales, las pesadas cargas de trabajo doméstico y la provisión de plantones para madera y postes en lugar de leña y frutas, productos que gozan de mayor preferencia.

En términos generales, los principales obstáculos que impiden la integración del género en las organizaciones y los programas forestales incluyen presupuestos insuficientes para incorporar el género junto con una limitada capacitación del personal en planificación y análisis de género.

Ante esta situación, varios individuos entrevistados propusieron algunas reformas para sus organizaciones: 

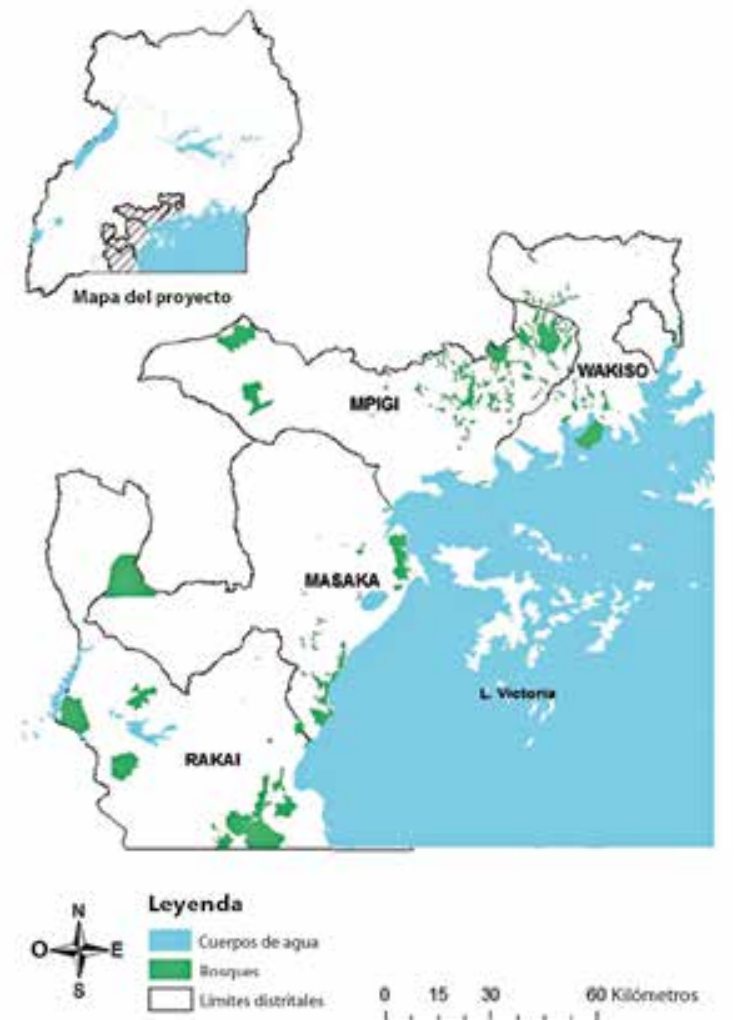

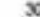
60 Kichetros

Figura 1. Región del lago Victoria incluyendo los distritos de estudio de Mpigi, Masaka y Rakai

- Otorgar incentivos al personal para que participe en el desarrollo de capacidades en las comunidades locales.

- Crear concientización entre el personal, especialmente en las posiciones de liderazgo, sobre el rol que las comunidades pueden desempeñar en la mejora del manejo forestal sostenible.

- Facilitar información a las comunidades locales en sus propias lenguas.

- Aumentar las redes de comunicación entre organizaciones que incentiven la participación de las comunidades locales.

Estas respuestas, aunque relevantes y útiles a nivel agregado, parecen confundir el género con las comunidades locales. Además, los individuos en las organizaciones encuestadas no vieron la necesidad de desagregar las comunidades por sexo (hombres y mujeres).

\section{El género en las asociaciones forestales (formales)}

Después de las reformas del 2001, algunas comunidades que viven junto a los bosques crearon asociaciones forestales para mejorar los medios de vida rurales. Más de dos tercios de ellas participan en acuerdos de Manejo Forestal Comunitario (MFC). Las asociaciones cercanas a los bosques privados participaron en el cultivo de árboles, comercial y en fincas, mientras que aquellas que vivían cerca de las reservas forestales locales, comunales y fuertemente degradadas, llevaron a cabo actividades de forestación y reforestación.

Las ocho asociaciones formales objeto de estudio son grupos de usuarios mixtos, aunque los hombres dominan las posiciones de liderazgo en seis de ellas. La mayoría de los grupos reportó una distribución equitativa de la mayor parte de los beneficios. Por ejemplo, los hombres y las mujeres pueden acceder de igual manera al apoyo técnico y financiero proporcionado por ONG e instituciones financieras. También tienen las mismas oportunidades para establecer contactos

\section{Cuadro 1. Estrategias organizativas para integrar el género}

$\%$ Respuesta $(\mathrm{n}=\mathbf{2 0})$

Organizaciones que toman en cuenta el 55

género durante la planificación estratégica

Organizaciones con planes operativos

que incluyen una clara asignación de

responsabilidades y tiempo para monitorear y

evaluar el impacto del género

Organizaciones que cuentan con una persona responsable de integrar el género

Personal de la organización que ha sido

capacitado en cuestiones de género

Personal de la organización que ha sido capacitado en la planificación y análisis de género

Capacitación de personal en cuestiones de género incluida en forma continua en el presupuesto

Presupuesto de la organización adecuado para apoyar la integración de género

Organizaciones que necesitan llevar a cabo reformas para integrar el género
40 con otros grupos de usuarios. Sin embargo, los hombres tienen mejores oportunidades de empleo en las actividades forestales organizadas por los grupos.

Si bien los hombres y las mujeres trabajan juntos la mayoría de las veces, también hay actividades que se llevan a cabo en función del género. Por ejemplo, los hombres se ocupan mayormente del cultivo de árboles de madera comercial en fincas y del patrullaje de los bosques, mientras que las mujeres tienen a su cargo principalmente el cultivo de árboles frutales y para leña en las fincas y la recolección de materiales para fabricar artesanías.

Todas las asociaciones informaron que la mayoría de miembros mujeres asistía regularmente a las reuniones, participaba plenamente y expresaba libremente sus opiniones. Aun así, en cerca del 30\% de los casos, los políticos locales y los miembros más acomodados controlaban la agenda, dominaban los debates e influían en la toma de decisiones.

\section{El género en los grupos de usuarios forestales informales}

Clasificamos a 10 comunidades, que vivían cerca de los bosques y no contaban con una asociación, como grupos de usuarios forestales informales. Estas comunidades obtienen prácticamente los mismos beneficios de los bosques que los grupos de usuarios formales y también distribuyen los beneficios por género de manera similar. Por ejemplo, las mujeres tenían a su cargo la administración de ocho viveros de plantones en el área de estudio.

La tenencia forestal y el género determinan los derechos y el acceso a los productos del bosque. En las reservas forestales del gobierno, tanto hombres como mujeres pueden obtener permisos para aprovechar legalmente productos forestales no maderables (PFNM). Los hombres son propietarios de los bosques privados y, a menudo, las mujeres no tienen acceso a estos recursos excepto para aprovechar leña (después de que los hombres hayan aprovechado la madera y los postes). Sin embargo, las mujeres pueden acceder fácilmente a los árboles de leña y los árboles frutales que crecen en las propiedades familiares. En los bosques plantados, los derechos de acceso están restringidos a los miembros de la familia. 
La directiva de la comunidad, incluidos los ancianos, controla el acceso a los bosques comunales en función de las normas culturales del área. Por lo general, estas instituciones tradicionales no incluyen mujeres en la toma de decisiones. Los bosques sagrados comunales son controlados por las personas ancianas del clan (que también excluyen a las mujeres) según los mitos y tradiciones de un bosque particular.

\section{El género en los consejos distritales y comités locales}

Los comités locales, creados por ley en 1987, fueron reforzados con la Ley de Gobiernos Locales de 1997. El comité de producción, por ejemplo, diseña políticas y controla todos los subsectores de producción en el distrito. La mayor parte de los encuestados fueron elegidos consejeros en los comités de producción a nivel de distrito y subdistrito. Otros encuestados pertenecían al personal técnico de entidades forestales y otras entidades gubernamentales que forman parte de los comités de producción como miembros no oficiales.

Los comités toman decisiones por consenso. Después de que el personal técnico explica los temas en detalle, los consejeros discuten libremente la moción. A todos los miembros, incluso a las mujeres, se les da la oportunidad de contribuir hasta que se llegue a un consenso. Sin embargo, en algunos casos, cuando se trata de asuntos conflictivos, se lleva a cabo una votación secreta. Los comités han tomado varias decisiones relacionadas con los bosques pero el cultivo de árboles es el tema que más atención ha recibido.

Todas las consejeras encuestadas reportaron haber influido en las decisiones del Consejo durante los últimos cinco años. Durante la temporada seca, por ejemplo, la única mujer en un Consejo convenció a sus colegas para destinar el dinero que se había aprobado originalmente para el ganado a los tanques de agua comunitarios. En otro caso, las consejeras convencieron al comité de producción de que observara el Día del Medio Ambiente sembrando árboles en tierras de propiedad de varias instituciones, incluyendo escuelas y centros religiosos.

\section{Lecciones aprendidas y recomendaciones}

Las pocas mujeres líderes que existen en proyectos y programas forestales no han sido capacitadas en cuestiones de género y no han realizado esfuerzos por mejorar la participación de las mujeres en el sector. Por ejemplo, durante la realización del estudio, solo 2 de los 110 servicios forestales distritales estaban encabezados por mujeres. La Reserva Forestal Central Mabira (Mabira CFR por sus siglas en inglés) cuenta en total con 14 trabajadores, de los cuales solo 4 son mujeres. De hecho, las mujeres que tienen cierto nivel de educación a menudo no son las mejores representando las necesidades de las mujeres: su estilo de vida, de la elite urbana, es muy diferente al de las mujeres rurales pobres y por ello carecen de un entendimiento profundo de las necesidades y prioridades de las mujeres rurales en el manejo forestal comunitario. Además, sus puestos son, con frecuencia, tan inseguros - los proyectos y programas son generalmente de corta duración con poca seguridad laboral- que no se atreven a "crear problemas". Por ello, existe a la necesidad de contar con una masa crítica de mujeres para poder abordar realista y exitosamente las cuestiones de género.

Aunque las mujeres participaron plenamente en las reuniones y toma de decisiones de la asociación de MFC, ellas tienden a participar menos en proyectos de cultivo de árboles y de MFC, que generalmente requieren más mano de obra y capital, que en los proyectos agroforestales de pequeña escala realizados desde las bases. Esto se atribuye al capital limitado, los largos periodos de rotación de los árboles maderables y las especies arbóreas menos preferidas que están siendo promovidas por las entidades del Gobierno, ONG y cultivadores de árboles.

Los principales obstáculos que impiden la participación de las mujeres en el cultivo de árboles en las fincas fueron las disparidades de género en la propiedad de la tierra, las normas y prácticas culturales, las pesadas cargas de trabajo doméstico y el limitado acceso a fuentes crediticias e insumos agrícolas, así como la provisión de plantones de especies arbóreas no necesariamente preferidas por las mujeres.

\section{Cuadro 2.Análisis de cuestiones de género en los distritos y propuestas para mejorar la participación de las mujeres}

\begin{tabular}{ll}
\hline $\begin{array}{l}\text { Preocupaciones clave en materia de } \\
\text { género }\end{array}$ & Efectos \\
\hline $\begin{array}{l}\text { Conocimiento y habilidades insuficientes } \\
\text { sobre cuestiones de equidad de género }\end{array}$ & $\begin{array}{l}\text { La asignación de recursos no aborda } \\
\text { las preocupaciones de mujeres y } \\
\text { hombres en forma equitativa }\end{array}$ \\
$\begin{array}{l}\text { Presupuesto y distribución de fondos } \\
\text { deficientes para incorporar las cuestiones de }\end{array}$ & $\begin{array}{l}\text { Pobre integración de género } \\
\text { géner }\end{array}$
\end{tabular}

deficientes para incorporar las cuestiones de género

La política de género revisada no ha sido suficientemente difundida dentro de los diferentes consejos gubernamentales a nivel local

Capacidad insuficiente para integrar el género en los subdistritos

Ausencia de personas específicas dedicadas al género (puntos focales) como lo estipula la ley a nivel de distrito y subdistrito

Poca contratación de mujeres en cargos de liderazgo político y técnico
Conocimiento escaso o inadecuado de las disposiciones de la política sobre género por parte de los consejeros en diferentes escalafones del gobierno local

Número limitado de personal técnico para liderar el proceso de incorporación de género a nivel de subdistrito

Implementación deficiente de las políticas y estrategias de género en los distritos

Marginación de los temas relacionados estructuras administrativas con las mujeres en políticas y

\section{Estrategias}

Capacitar a los jefes de sectores, departamentos y líderes políticos sobre cuestiones de género, incluyendo la equidad en la elaboración de presupuestos

Aumentar la asignación presupuestaria destinada al programa de género

Difundir la política de género de Uganda entre el personal técnico y los líderes políticos a nivel de distrito y subdistrito Distribuir copias de la política a todos los departamentos de los diferentes sectores

Traducir la política a las lenguas locales

Asegurar la participación del personal de distrito y subdistrito en la generación y uso de los datos desagregados por género Desarrollar herramientas para la recolección de datos

Capacitar a los encargados del desarrollo comunitario en el análisis de género

Cubrir posiciones de género vacantes a nivel de distrito y subdistrito

Aumentar las oportunidades para que las mujeres ocupen cargos políticos y administrativos mediante la acción afirmativa 
Las mujeres estaban representadas en los grupos de usuarios forestales mixtos formales. Ellas participaron activamente en las actividades, guiadas por las constituciones de la asociación que exigen la inclusión de las mujeres en posiciones de liderazgo. La existencia de una constitución negociada por todos los actores, incluyendo las mujeres, las empodera y reduce las disparidades de género en las áreas de información y concientización. Por ello, la calidad y nivel de la participación de las mujeres en la toma de decisiones en el manejo forestal parecen haber mejorado cuando las mujeres se incorporan a los grupos de usuarios forestales formales. Además, la presencia de un gran número de mujeres en las asociaciones les permite discutir y expresar sus opiniones libremente.

La participación de las mujeres en la toma de decisiones y las actividades de las asociaciones forestales podría mejorarse si:

- Un mayor número de mujeres asume posiciones de liderazgo en las asociaciones de usuarios forestales formales de manera que puedan diseñar mejor la agenda.

- Se establecen más ONG con estrategias afirmativas a nivel de las bases.

- $\quad$ Se establecen más contactos entre ONG, no solo para evitar la duplicación de actividades sino también para mejorar la capacidad de las pequeñas ONG locales que no cuentan con personal capacitado en cuestiones de género.

- Se establecen más contactos e intercambios entre asociaciones forestales de manera que puedan aprender de sus experiencias.

En los tres distritos estudiados, las mujeres ocupaban el 5\% de los puestos técnicos y de liderazgo político. Este nivel bajo de representación afecta la capacidad de las mujeres de diseñar la agenda e influir en la toma de decisiones del Consejo. Por ello, existe la necesidad de establecer medidas de acción afirmativa en materia de género tanto a nivel de distrito como de subdistrito.

La ausencia de personas dedicadas a materias de género (puntos focales), como lo estipula la ley, dificulta la implementación de una política de género en los distritos. A nivel de subdistrito, la mayor parte de plazas para el puesto de encargado de desarrollo comunitario están vacantes, mientras que a nivel de distrito, los mismos puestos en Masaka y Mpigi estaban ocupados por funcionarios que también se desempeñaban en otras carteras. Esto demuestra que existe poco personal técnico con capacidad para liderar el proceso de integración de género a nivel de distrito y subdistrito. Recomendamos que el puesto de encargado de desarrollo comunitario en todos los niveles sean llenados y que los funcionarios sean capacitados en análisis de género de manera que puedan recabar datos de género desagregados para la planificación, el desarrollo de capacidades y la elaboración de presupuestos.

Es necesario que el Ministerio de Género, Trabajo y Asuntos Sociales, así como las ONG con proyectos desde las bases en materia de género, difundan la política de género de Uganda entre el personal técnico y los líderes políticos a nivel de distrito y subdistrito. Además, el Ministerio debería capacitar a este mismo grupo en cuestiones de género, incluyendo la equidad en los presupuestos.

\section{Agradecimientos}

Quisiéramos agradecer a todas las personas que colaboraron con nosotros proporcionándonos información para el estudio: personal ejecutivo y administrativo de ONG, funcionarios del Gobierno en ministerios y agencias y los Oficiales Forestales de Distrito en Mpigi, Masaka y Rakai. También queremos expresar nuestro reconocimiento a D. Waiswa, de la Facultad de Ciencias Forestales, Biodiversidad y Turismo, por preparar el mapa de estudio. Asimismo deseamos manifestarle nuestra gratitud a la Agencia Austríaca para el Desarrollo (ADA) por proporcionarnos los fondos y a CIFOR (Esther Mwangi y Anne Larson) por administrar y orientar la investigación. También queremos agradecerle a AUPWAE, nuestros socios en este proyecto, por su gran liderazgo. Por último, deseamos expresarle nuestro reconocimiento a Mark Foss por la edición del documento.

\section{Referencias}

Banana, A. Y., Buyinza, M., Luoga, E. y Ongugo, P. 2010 Emerging local economic and social dynamics shaping East African forest landscapes. En: Guido, M., Katila, P., Alfaro, R. I., Kanninen, M., Labovikov, M. y Varjo, J. (eds.) Forests and Society - responding to global drivers of change, IUFRO World Series 25: 315-335. Global Network for Forest Science Cooperation, Viena.

Howard, P. L. y Nabanoga, G. 2007 Are there customary rights to plants? An inquiry among the Baganda (Uganda), with special attention to gender. World Development 35 (9): 1542-1563.

Mwangi, E., Meinzen-Dick, R. y Sun, Y. 2011 Gender and sustainable forest management in East Africa and Latin America. Ecology and Society 16(1): 17-25.

Obua J., Banana, A. Y. y Turyahabwe, N. 1998 Attitudes of local communities towards forest management practices in Uganda: the case of Budongo forest reserve. Commonwealth Forestry Review 77(2): 113-118.

Esta investigación fue realizada por CIFOR como parte del Programa de Investigación de CGIAR sobre Bosques, Árboles y Agroforestería. El objetivo del programa es mejorar el manejo y uso de los bosques, la agroforestería y los recursos genéticos de los árboles a lo largo del paisaje, desde bosques hasta plantaciones. CIFOR dirige el programa de colaboración en asociación con Bioversity International, CIRAD, el Centro Internacional de Agricultura Tropical y el Centro Mundial de Agroforestería.

\section{Austrian \\ $=$ Development Agency}

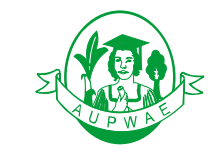

